\title{
Hernia de Petit congénita en un escolar. Reparación con malla protésica. Reporte de caso
}

\author{
Roberto Martínez-Quiroz ${ }^{1}$, Luis Enrique Sánchez-Sierra², David Alexander Montoya-Reales ${ }^{2}$, \\ Josue F. Rodas-Andino ${ }^{2}$ y Andrés Eduardo Velásquez-Bueso ${ }^{2}$.
}

Congenital Petit's hernia in a Escolar. Prosthetic Mesh Repair.

\section{Case Report}

Introduction: The abdominal wall congenital lumbar hernia is a rare condition, There are around 50 cases describe in the English literature. These are classified according to their anatomical site of appearance, upper or lower lumbar triangle. Case presentation: A 6-year-old male patient presented congenital left mass in the lumbar region, reductible, size $10 \times 8 \mathrm{~cm}$; during abdominal ultrasound, hernia was compatible with Petits congenital hernia. A reduction of the hernial sack with anterior plasty and placement of the prosthetic mesh at the defect site was performed. Discussion: The triangle of Grynfelt-Lesshaft, is larger and more constant than the triangle of Petit, the latter represents the least common location. Several repair techniques are described, but anterior hernioplasty is the most recommended. Conclusion: Lumbar hernia should be considered as a differential diagnosis in all newborns with protruding mass on the left or right flank at birth, accompanied or not by another obvious or non-evident malformation. Early diagnosis avoids possible complications and allows for opportune treatment, allowing the patient a better lifestyle. Key words: congenital lumbar hernia; Petit's hernia; posterolateral abdominal wall.

\section{Resumen}

Introducción: La hernia lumbar de pared abdominal congénita es una condición rara, existen alrededor de 50 casos publicados en la literatura inglesa; se clasifican según su sitio anatómico de aparición: en triángulo lumbar superior e inferior. Caso clínico: Paciente masculino, 6 años de edad, presenta masa en región lumbar izquierda, congénita, reducible, tamaño de 10 × $8 \mathrm{~cm}$. El ultrasonido abdominal mostró defecto herniario compatible con hernia de Petit congénita. Se realizó reducción del saco herniario con plastía anterior y colocación de malla protésica en el defecto. Discusión: El triángulo de Grynfelt-Lesshaft es más grande y constante que el de Petit, este último representa el lugar menos común de localización. Se describen varias técnicas de reparación, pero la hernioplastía anterior es la más recomendada. Conclusiones: La hernia lumbar debe considerarse como diagnóstico diferencial en todo recién nacido que presenta masa en flanco izquierdo o derecho al nacimiento, con presencia o ausencia de otra malformación. El diagnóstico temprano evita complicaciones y permite el tratamiento oportuno, ofreciendo una mejor calidad de vida al paciente.

Palabras clave: hernia lumbar congénita; hernia de Petit; pared abdominal posterolateral.

\section{Introducción}

Las hernias de pared abdominal poco frecuentes incluyen: Hernía de Spiegel, obturatriz, lumbares e intraparietales ${ }^{1}$. Las hernias lumbares (HL) se conocen desde 1672, sugeridas por Barbette y confirmadas en 1731 por De Garengeot durante una autopsia ${ }^{2}$.

La HL es un defecto de la pared abdominal posterolateral poco frecuente ${ }^{3}$. La hernia lumbar congénita (HLC) se asocia con anomalías congénitas como costillas, músculos, riñones y meninges. Menos de 50 casos de HLC asociada a otra anomalía congénita se ha publicado, siendo una entidad muy rara $^{4}$, y se
Servicio de Cirugía Pediátrica, Hospital Escuela Universitario. ¿Universidad Nacional Autónoma de Honduras. Tegucigalpa, Honduras.

Recibido 18 de abril de 2018 y aceptado para publicación el 28 de mayo de 2018.

Correspondencia a: Dr. Luis Enrique SánchezSierra

luensasi90@hotmail.com 
Figura 1. Hernia de Petit izquierda. La hernia protruye de manera espontánea durante la inspiración y con la maniobra de Valsalva.

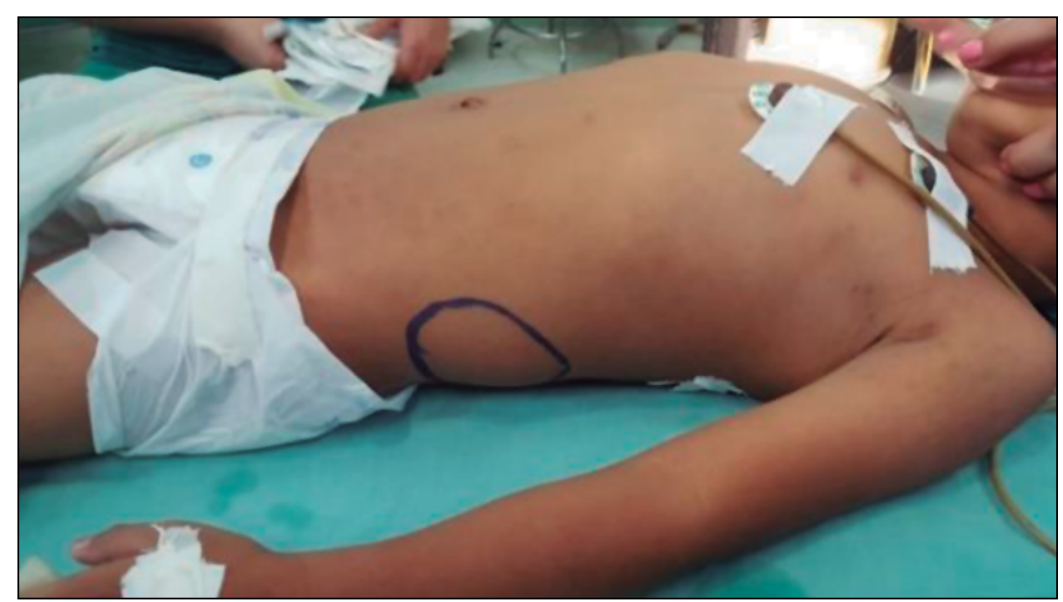

Figura 2. Hernia de Petit izquierda, previo a la cirugía. Paciente en decúbito supino, se marcó la zona del defecto de la pared abdominal, previo a la cirugía.

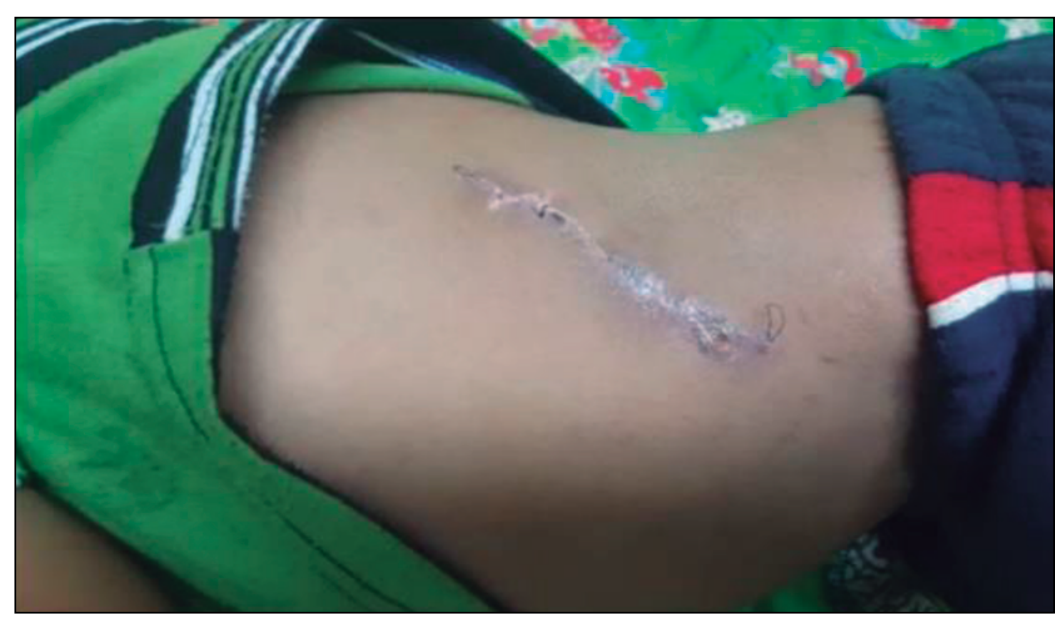

Figura 3. Cicatriz de herida quirúrgica. El paciente fue evaluado 15 días posoperatorio para el retiro de hilo de sutura de la herida quirúrgica. han descrito alrededor de 300 casos de HL en la literatura médica mundial ${ }^{5}$. La HL representa $1,5-2 \%$ de las hernias abdominales ${ }^{6}$.

El espacio lumbar tiene dos zonas débiles donde se puede producir la HL: triángulo lumbar superior o de Grynfelt-Lesshaft y triángulo lumbar inferior o de Petit ${ }^{7}$, pero se localizan con mayor frecuencia en el triángulo lumbar superior izquierdo ${ }^{3}$.

El caso presentado en este artículo corresponde a una hernia lumbar de Petit en un infante, caso poco frecuente, que sirve de base para el análisis y traer a acotación la existencia de estas hernias, lo cual debe estar presente en el abánico de posibilidades diagnósticas que maneja el cirujano.

\section{Caso clínico}

Paciente masculino de 6 años de edad, se presentó con una masa en pared abdominal posterior lateral, en flanco izquierda, padre del paciente refirió que el defecto se identificó desde el nacimiento, ésta protruye con maniobras de Valsalva y espontáneamente con movimientos respiratorios, no presenta cambios de color sugestivo de inflamación, se auscultó ruidos intestinales, abdomen timpánico, se palpó en flanco izquierdo una masa ovalada en posición oblicua, no dolorosa, reducible, de bordes regulares, anillo herniario delimitado con $3 \mathrm{~cm}$ de diámetro y saco herniario $10 \mathrm{x} 8 \mathrm{~cm}$; la masa no limitaba sus actividades diarias (Figura 1). El ultrasonido abdominal, mostró defecto herniario en región lumbar izquierda, saco herniario contenía asas de intestinales delgado, hernia no complicada. El paciente presentó, además, polidactilia posaxial y articulada en la mano derecha.

Paciente tuvo nacimiento a término por cesárea debido a baja reserva fetal, hubo seguimiento cada año por consulta externa de cirugía pediátrica del Hospital Escuela Universitario de Tegucigalpa, se evaluó a los 6 años, como tiempo de espera para reducción espontánea de la hernia. En ese momento se tomó la decisión de realizar intervención quirúrgica (Figura 2). Se realizó reducción del saco herniario con plastía anterior y colocación de malla protésica en el sitio del defecto. Durante la cirugía, la hernia se encontró limitada en el triángulo lumbar inferior, anillo herniario de $5 \mathrm{~cm}$ de diámetro y el saco herniario tenía contenido de asas de intestino delgado y grasa retroperitoneal, no complicada.

La recuperación posoperatoria fue satisfactoria y 15 días poscirugía se realizó retiro del hilo de sutura, no mostró recurrencia (Figura 3).

\section{Discusión}


El triángulo lumbar superior o de GrynfeltLesshaft, tiene forma invertida limitado en su base por la $12^{\mathrm{a}}$ costilla; borde inferior, el músculo serrato posteroinferior; lado posterior, el músculo sacroespinal; anterior el músculo oblicuo interno; techo músculo oblicuo externo y dorsal ancho; el suelo es la fascia transversalis y la aponeurosis del músculo transverso. El triángulo lumbar inferior o de Petit, tiene como límites la cresta del hueso iliaco como base; el músculo oblicuo externo como borde lateral y músculo dorsal ancho como borde medial; el suelo lo forma la fascia lumbodorsal ${ }^{7,8}$. De acuerdo con las características anatómicas, este caso es compatible con la hernia de Petit congénita.

La HL es una entidad rara, el triángulo de Grynfelt-Lesshaft es más grande y constante que el triángulo de Petit, este último representa el lugar menos común de localización ${ }^{3,9}$. La HL superior e inferior se presenta con mayor frecuencia en varones, entre 50 y 70 años, en el lado izquierdo ${ }^{10}$. Estas hernias pueden ser congénitas en $20 \%$ y adquiridas en $80 \%$ de los $\operatorname{casos}^{7,11}$. En el presente caso, hablamos de una hernia con características pocos frecuentes, ya que es un escolar de 6 años, cuya etiología aún se desconoce, coincidiendo en 2 parámetros de frecuencia: varón y lado izquierdo.

La HL adquirida puede ser primaria o secundaria, las secundarias son traumáticas o posquirúrgicas en el $25 \%$ de $\operatorname{casos}^{8}$, se ha observado que las hernias congénitas surgen del triángulo inferior y a menudo se observan con otras anomalías como la agenesia renal y síndrome lumbocostovertebral ${ }^{9,12}$. En este caso la hernia se asoció únicamente con polidactilia posaxial articulada derecha, esta relación no se encontró en ningún otro caso de HL publicado, podríamos definirlo como primer reporte de presentación clínica, cuya relación debe ser estudiada.

La hernia difusa es una tercera variedad de HL, representa el $5 \%$ de los $\operatorname{casos}^{11}$. El contenido de la hernia lumbar puede ser grasa retroperitoneal, riñón, colon, intestino delgado, epiplón, ovario, bazo o apéndice ${ }^{8,13}$. En el presente caso el contenido fue asas de intestino delgado, identificadas mediante ultrasonido (USG) abdominal, pero con reducción espontánea, durante la cirugía debido a la relajación y disminución de la presión intrabdominal, el volumen del saco herniario era menor, siempre conteniendo asas de intestino delgado y grasa retroperitoneal.

El diagnóstico diferencial debe incluir tumores renales, contracturas musculares, tumores de partes blandas (lipoma), hematomas y abscesos ${ }^{8,10}$. El estándar de oro diagnóstico, es la tomografía computarizada, esta permite distinguir las capas de músculo y fascia, distingue defectos en una o más capas de la pared, distingue entre hernia y tumor y evalúa contenido herniario ${ }^{11}$. En este caso el USG abdominal permitió el diagnóstico definitivo debido a las características bien definidas de anillo, saco y contenido herniario.

Se sugiere, una hernioplastía anterior en los pequeños defectos de contenido extraperitoneal; se sugiere la vía laparoscópica transabdominal en defectos moderados con hernias intraperitoneales y en las recidivas o hernias difusas con tamaño superior a $10 \mathrm{~cm}$, una plastía anterior con doble malla ${ }^{14}$. El estudio de Moreno-Egea en 2017 establece que la reparación de cualquier hernia lumbar, independiente de tamaño y localización, precisará cubrir todo el espacio oval, para garantizar su seguridad ${ }^{13}$. En nuestro caso se realizó reducción del saco herniario con plastía anterior y colocación de malla protésica fijada a la fascia, cubriendo todo el sitio del defecto, el paciente fue evaluado un año después de la hernioplastía y no presentó recidiva. La malla utilizada en este paciente fue de monofilamento de polipropileno, debido que es con la que contamos en nuestro servicio hospitalario.

La HL es poco frecuente y la hernia de Petit congénita es muy rara, pero debe tenerse presente como diagnóstico diferencial en todo recién nacido que presenta masa en flanco izquierdo o derecho al nacimiento, acompañado o no de otra malformación. El diagnóstico temprano evita posibles complicaciones y permite un tratamiento oportuno, garantizando al paciente un mejor estilo de vida.

\section{Responsabilidades éticas}

Protección de personas y animales. Los autores declaran que para esta investigación no se han realizado experimentos en seres humanos ni en animales.

Confidencialidad de los datos. Los autores declaran que en este artículo no aparecen datos de pacientes.

Conflictos de interés: no hay. 


\section{Referencias}

1. Satorras-Fioretti AM, Vázquez-Cancelo J, Pigni-Benzo L, Salem AM, RamosArdá A. Hernias de pared abdominal de localización poco frecuente. Cir Española 2006;79:180-3.

2. González Rodríguez FJ, Gómez AP, López M, Conde Freire RF, González Vinagre S, Barreiro Morandeira F, Bustamante Montalvo M. Hernia de Grynfelt. Discusión y manejo. Rev Hispanoam Hernia 2014;2:63-6.

3. Cesar D, Valadao M, Murrahe RJ. Grynfelt hernia: Case report and literature review. Hernia 2012;16:107-11.

4. Rattan KN, Agarwall A, Dhiman A, Rattan A. Int J Pediatr. 2016;1:1-4

5. Mismar A, Al Ardah M, Albsoul N, Younes N. Underlay Mesh repair for spontaneus lumbar hernia. Int J Surg. 2013;4:534-6.
6. Ploneda-Valencia CF, Cordero-Estrada E, Castañeda-González LG, SainzEscarrega VH, Varela-Muñoz O, De la Cerda-Trujillo LF, et al. Grynfelt-lesshaft hernia a case report and review of the literatura. Annals of medicine and Surgery 2016;7:104-6.

7. García Calleja JL. Hernia lumbar superior adquirida espontánea: Hernia de GrynfeltLesshaft. Cir Esp. 2015;93:718.

8. Sundaramurthy S, Suresh HB, Anirudh AV, Prakash Rosario A. Primary lumbar hernia: A rarely encountered hernia. Int $\mathbf{J}$ Surg. 2016;20:53-6.

9. Gupta L, Mala TA, Gupta R, Malla SA. Lumbo-Costo-Vertebral Syndrome With Congenital Lumbar Hernia. J Case Rep. 2014;5:5

10. Orozco Gil N, Martínez Ballester ML, Bañuls Matoses A, Montesinos Meliá C, Caro Pérez F. Hernia de Petit incarcerada: a propósito de un caso. Rev Hispanoam Hernia 2016;4:33-6.

11. Suárez S, Hernández JD. Laparoscopic repair of a lumbar hernia: report of a case and extensive review of the literature. Surg Endosc. 2013;27:3421-9.

12. Sharma P. Lumbar hernia. Med J Armed Force India. 2009; 65:178-9.

13. Moreno-Egea A. Hernia lumbar, espacio oval y reconstrucción de la pared abdominal posterior. Rev Hispanoam Hernia 2017;5:95-9.

14. Moreno-Egea A. Hernias lumbares. Hernias de Spiegel. Hernia obturatriz. En: Morales Conde S, Barreiro Morandeira F, Hernández Granados $\mathrm{P}$, Feliu Palà X, editores. Guías clínicas de la Asociación Española de Cirujanos. Cirugía de la pared abdominal. $2^{\mathrm{a}} \mathrm{ed}$. Madrid: Grupo ARÁN ediciones; 2012. pp. 241-52. 